International Journal of Modern Physics A,

(C) World Scientific Publishing Company

\title{
NEW INSIGHTS CONCERNING DIMENSION EIGHT EFFECTS IN WEAK DECAYS
}

\author{
JOHN F. DONOGHUE \\ Physics Department, University of Massachusetts, Amherst MA 01003
}

\begin{abstract}
Most past work on weak nonleptonic decays has mixed dimensional regularization in the weak operator product expansion with some form of a cutoff regularization in the evaluation of the matrix elements. Even with the usual technique of matching the two schemes, this combination misses physics at short distance which can be described by dimension eight (and higher dimension) operators. I describe some recent work with V. Cirigliano and E. Golowich which clarifies these effects and provides a numerical estimate suggesting that they are important.
\end{abstract}

\section{Introduction}

Weak nonleptonic decays are especially difficult to calculate. The short distance nature of the $\mathrm{W}$ propagator implies that all values of the momentum from low energy up to the W mass contribute to the process. Because we don't have a satisfactory method to calculate all these scales at once, we attack the problem in parts, using two ideas originally due to Ken Wilson - the separation of scales and the operator product expansion. We first imagine separating the problem into short distance physics and long distance physics, with the separation scale being called $\mu$. When we look at the short distance physics, we see the weak current product modified by the radiative corrections of quarks and gluons. Since all aspects are at short distance we can represent the effect of these corrections as local operators. This brings in the operator product expansion, with the effective weak Hamiltonian at this scale being given by a sum over a complete set of operators with coefficients calculable in QCD perturbation theory. However, we then need to add back in the long distance physics by taking the matrix elements of the operators including physics of energies up to the scale $\mu$. Such matrix elements cannot use perturbation theory but are best accomplished with low energy hadronic methods. This separation of the physics into long and short distance regions using the OPE allows us to use the best methods available for each region.

The above description is what we as a field have always thought that we were doing both in theory and in practice. However, in fact most calculations were not doing this. The departure from the above description comes in the use of dimensional regularization in the calculation of the OPE coefficients. Dimensional regularization does not provide a complete separation of scales. The parameter $\mu$ that appears in dimensional regularization does not function as a boundary between short and long distance. In practice, only the most singular pieces of short distance physics is captured when regularizing dimensionally. This is sufficient to regularize the singular local operators of the OPE and to sum the large logarithms of the W mass. But it does not provide the complete Wilsonian integrating-out of all short 
distance physics. Some less singular physics remains to be included in the physical amplitudes.

Of course, dimensional regularization is a perfectly fine scheme to use in the calculation, if employed consistently. But in practice most calculations mix regularization schemes, using some form of a cutoff to calculate matrix elements. Thus the matrix elements are done with a rigorous separation of scales, while the coefficients are not. This is not consistent. What is missing from the final answer is the less singular short distance physics from the short distance parts of the calculation.

This conflict can be phrased in terms of the operators that appear on the OPE. Dimensional regularization involves operators of dimension six in the OPE. The less singular short distance physics can be described by operators of dimension eight and higher. The question is where the physics of dimension eight appears. Present practice must be modified in one of the following ways:

- If one calculates the matrix elements including physics only up to the scale $\mu$, one must include dimension-eight operators in the OPE with coefficient of order $1 / \mu^{2}$.

- If instead one wants to use dimensional regularization throughout, one must include physics of all scales in the matrix elements. The high energy portion of the matrix element above the scale $\mu$ is the effect of dimension-eight (and higher) operators.

Our paper ${ }^{1}$ provides a concrete demonstration of both of these options and a calculation of the dimension eight operators relevant for the Standard Model at one loop. Here I can merely sketch the flow of this demonstration.

We calculate a particular type of weak matrix element that through some manipulation can be written as the integral over vector and axial vacuum polarization functions.

$$
\mathcal{M}=\frac{3 G_{F} M_{W}^{2}}{32 \sqrt{2} \pi^{2} F_{\pi}^{2}} \int_{0}^{\infty} d Q^{2} \frac{Q^{4}}{Q^{2}+M_{W}^{2}}\left[\Pi_{V, 3}\left(Q^{2}\right)-\Pi_{A, 3}\left(Q^{2}\right)\right] .
$$

Here the hadronic information is contained in the vacuum polarization functions. We can pick out all the ingredients to the amplitude by studying this function.

First we will consider the case where we separate the physics above and below some value of $Q^{2}=\mu^{2}$. The high energy parts of the vacuum polarization can be written (in the chiral limit) in terms of operators of dimension-six, dimension-eight and higher. The specific form is not too interesting here, but the reader should just follow the superscript to the operator, which indicates the dimension. Thus

$$
\left(\Pi_{V, 3}-\Pi_{A, 3}\right)\left(Q^{2}\right) \sim \frac{2 \pi\left\langle\alpha_{s} \mathcal{O}_{8}^{(6)}\right\rangle_{\mu}}{Q^{6}}+\frac{\mathcal{E}_{\mu}^{(8)}}{Q^{8}}+\ldots .
$$

To complete the calculation in the scheme where we fully separate the scale we define a hadronic matrix element by including all physics up to an energy cutoff at 
the scale $\mu$. We end up finding

$$
\mathcal{M} \simeq \frac{G_{F}}{2 \sqrt{2} F_{\pi}^{2}}\left[\left\langle\mathcal{O}_{1}^{(6)}\right\rangle_{\mu}^{(\text {c.o. })}+\frac{3}{8 \pi} \ln \left(\frac{M_{W}^{2}}{\mu^{2}}\right)\left\langle\alpha_{s} \mathcal{O}_{8}^{(6)}\right\rangle_{\mu}+\frac{3}{16 \pi^{2}} \frac{\mathcal{E}_{\mu}^{(8)}}{\mu^{2}}+\ldots\right] .
$$

where the first two operators are of dimension six. This is the correct OPE for this matrix element in the case where we fully separate the short distance from long distance physics. In this case the dimension eight operator appears in the OPE and has come for the sub-leading effect at short distance.

On the other hand we can go back and define the local operator not by a cutoff but by dimensional regularization. This results in a definition in which all momentum scales are present

$$
\left\langle\mathcal{O}_{1}^{(6)}\right\rangle_{\mu_{\text {d.r. }}}^{(\text {d.r. }}=\frac{(d-1) \mu_{\text {d.r. }}^{4-d}}{(4 \pi)^{d / 2} \Gamma(d / 2)} \int_{0}^{\infty} d Q^{2} \quad Q^{d}\left(\Pi_{V, 3}-\Pi_{A, 3}\right)\left(Q^{2}\right)
$$

Even without evaluating the integral we can see that to know its value we must include physics from above the scale $\mu_{\text {d.r. }}$, since there is no separation of scales. After

removing the divergences in the $\overline{\mathrm{MS}}$ scheme, we can then obtain the renormalized operator

$$
\left\langle\mathcal{O}_{1}^{(6)}\right\rangle_{\mu_{\text {d.r. }}}^{\overline{\mathrm{MS}}}=\left\langle\mathcal{O}_{1}^{(6)}\right\rangle_{\mu}^{(\text {c.o. })}+\frac{3 \alpha_{s}}{8 \pi}\left[\ln \left(\frac{\mu_{\text {d.r. }}^{2}}{\mu^{2}}\right)-\frac{1}{6}\right]\left\langle\mathcal{O}_{8}^{(6)}\right\rangle_{\mu}+\frac{3}{16 \pi^{2}} \frac{\mathcal{E}_{\mu}^{(8)}}{\mu^{2}} .
$$

The mixing of dimension six operators is expected in a typical matching situation. However, we also see that dimension eight operators need to be included in the matching between a cutoff scheme and dimensional regularization. This is what is normally missed. It comes from the part of the integral that is above the cutoff, i.e. from short distance physics. The particular size of the dimension eight component in the operator is exactly the same as was found in the OPE above. Therefore the OPE in a dimensional scheme can be done with only dimension-six operators in the OPE. The dimension eight effect is in the matrix element instead.

Within this calculation, we can also calculate reliably the magnitude of the dimension eight effect. This is because the vacuum polarization functions satisfy dispersion relations with the input being given by data on $e^{+} e^{-}$reactions and $\tau$ decays. What we find is that the dimension eight correction is $100 \%$ of the leading dimension six operator when $\mu \sim 1.5 \mathrm{GeV}$, and scales as $1 / \mu^{2}$.

Most calculations of $\epsilon^{\prime} / \epsilon$ reported in the literature were done with scales at 1 $\mathrm{GeV}$ or below. These dimension eight effects (and even higher dimension effects) certainly would be present. These constitute an extra uncertainty in the predictions.

\section{References}

1. V. Cirigliano, J. F. Donoghue and E. Golowich, "Dimension-eight operators in the weak OPE," hep-ph/0007196. 